ALEA, Lat. Am. J. Probab. Math. Stat. 15, 1-20 (2018)

DOI: 10.30757/ALEA.v15-01

\title{
Concentration results for directed polymer with unbounded jumps
}

\author{
Shuta Nakajima \\ Research Institute in Mathematical Sciences, Kyoto University, Kyoto, Japan \\ E-mail address: njima@kurims.kyoto-u.ac.jp
}

\begin{abstract}
We study the free energy and the ground state energy for the directed polymer in random environment. The polymer is allowed to make unbounded jumps and the environment is given by i.i.d. Bernoulli variables. Our goal is to derive concentrations for the ground state energy of polymers at zero temperature and the free energy at any temperature. To do this, we control the maximum jump of any polymer nearly minimizing energy and guarantee that it is not too large with high probability.
\end{abstract}

\section{Introduction}

We discuss the models of directed polymer which have unbounded jumps that were introduced in Comets et al. (2015). See Comets et al. (2015) for the background and related works. That paper exhibits the following three results: (i) The continuity of the free energy with respect to inverse temperature and the appearance probability of obstacles; (ii) The asymptotic of the free energy as the appearance probability of obstacles goes to 1; (iii) The continuity of the time constant of First Passage Percolation (FPP) related to the model. In this paper, we show concentration bounds for the ground state energy, which is nothing but the minimum passage time, and the free energy. As applications, we derive the so-called "rate of convergence" results and, based on them, we are able to remove the restriction left in Comets et al. (2015) on a parameter in (i) and also give an alternative proof of (iii).

In fact, a concentration bound for the lower tail for the FPP is shown in Proposition 3.1 of Comets et al. (2015). However the upper tail is significantly more difficult as we need to control the maximum jump of the optimal path. Although such a geometric property of the minimizing path is usually hard to prove, in this paper, we succeed in controlling jumps not only of the optimal path but also of

Received by the editors November 18th, 2016; accepted December 9th, 2017.

2010 Mathematics Subject Classification. Primary 60K37; secondary 60K35; 82A51; 682D30.

Key words and phrases. Directed polymer, random environment, first passage percolation, ground states, zero temperature. 
low energy paths in the directed polymer model. As a result, we can show the concentration for the minimum passage time and the free energy. The bound on the maximum jump itself is of independent interest, see Corollary 2.7 for the precise statement.

Let us briefly explain the motivation of Comets et al. (2015) and this work. There has been revived interest on the number of open paths in oriented percolation, e.g. Fukushima and Yoshida (2012) and Garet et al. (2017). It can be regarded as a zero temperature limit of the directed polymer in random environment and hence the free energy, which corresponds to the growth rate of the number of open paths, is expected to be continuous at zero temperature. Unfortunately, there seems to be few techniques developed to study the zero temperature limit in this type of models. In Comets et al. (2015), we introduced a toy model where the polymer is allowed to make unbounded jumps and established the continuity at zero temperature by a combinatorial argument. This paper proposes alternative arguments for the continuity based on the concentration estimates.

1.1. Setting of models. Let $\left(\left\{X_{n}\right\}_{n \in \mathbb{N} \cup\{0\}}, P\right)$ be the random walk on $\mathbb{Z}^{d}$ starting from 0 and with the transition probability

$$
P\left(X_{n+1}=z \mid X_{n}=y\right)=f_{\alpha}\left(|y-z|_{1}\right),
$$

where $|x|_{1}:=\sum_{i=1}^{d}\left|x_{i}\right|$ for $x \in \mathbb{Z}^{d}$ and $f_{\alpha}: \mathbb{N} \cup\{0\} \rightarrow \mathbb{R}$ is a function of the form

$$
f_{\alpha}(k)=c_{1} \exp \left\{-c_{2} k^{\alpha}\right\},
$$

where $\alpha, c_{2}>0$ and $c_{1}$ is a positive constant determined as to be $\sum_{y \in \mathbb{Z}^{d}} f_{\alpha}\left(|y|_{1}\right)=1$ (see Remark 1.9 for generalizations). The random environment is modelled by independent and identically distributed Bernoulli random variables

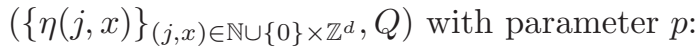

$$
Q(\eta(0,0)=1)=p \in(0,1) .
$$

We introduce the Hamiltonian

$$
H_{n}^{\eta}(X)=\sum_{j=1}^{n} \eta\left(j, X_{j}\right),
$$

and define the partition functions by

$$
Z_{n}^{\eta, \beta}=P\left[\exp \left\{\beta H_{n}^{\eta}\right\}\right] \text { for } \beta \in \mathbb{R} \text { and } Z_{n}^{\eta,-\infty}=P\left(H_{n}^{\eta}=0\right),
$$

where $P[\cdot]$ denotes the expectation with respect to $P$. Note that $Z_{n}^{\eta,-\infty}$ is positive for $Q$-almost every $\eta$, since the random walk has unbounded jumps.

An important quantity in this model is the so-called free energy defined by

$$
\begin{aligned}
\varphi(p, \beta) & =\lim _{n \rightarrow \infty} \frac{1}{n} \log Z_{n}^{\eta, \beta} \\
& =\lim _{n \rightarrow \infty} \frac{1}{n} Q\left[\log Z_{n}^{\eta, \beta}\right]
\end{aligned}
$$

$Q$-almost surely, whose existence can be shown by using the subadditive ergodic theorem. In Comets et al. (2015), the continuity property and some asymptotic behavior of the free energy were studied. 
Next, we introduce the FPP models related to this directed polymer model. Let us define a point process representing the sites where $\eta$ takes value zero by

$$
\omega_{p}=\sum_{(k, x) \in \mathbb{N} \times \mathbb{Z}^{d}}(1-\eta(k, x)) \delta_{\left(k, s_{p} x\right)},
$$

with the scaling factor $s_{p}=(1-p)^{1 / d}$. This scaling is natural since $\omega_{p}$ converges as $p \uparrow 1$ to the Poisson point process $\omega_{1}$ on $\mathbb{N} \times \mathbb{R}^{d}$ whose intensity is the product of the counting measure and the Lebesgue measure. With some abuse of notation we will frequently identify $\omega_{p}$, and more generally any point measure, with its support. Given a realization of $\omega_{p}$, we define the minimum passage time from 0 to $n$ by

$$
T_{n}\left(\omega_{p}\right)=\min \left\{\sum_{k=1}^{n}\left|x_{k-1}-x_{k}\right|_{1}^{\alpha}: x_{0}=0 \text { and }\left\{\left(k, x_{k}\right)\right\}_{k=1}^{n} \subset \omega_{p}\right\} \text {. }
$$

This is the directed version of Howard-Newman's Euclidean FPP model in Howard and Newman (2001). Now, a direct application of the subadditive ergodic theorem shows that the limit

$$
\mu_{p}=\lim _{n \rightarrow \infty} \frac{1}{n} T_{n}\left(\omega_{p}\right)=\lim _{n \rightarrow \infty} \frac{1}{n} Q\left[T_{n}\left(\omega_{p}\right)\right]
$$

exists $Q$-almost surely. The limit $\mu_{p}$ is non-random and called the time constant. Observe also that definition (1.4) makes perfect sense when $p=1$, yielding a limit $\mu_{1}$ in (1.5). It is again shown in Comets et al. (2015) that $\mu_{p}$ is continuous as $p \uparrow 1$.

This FPP is related to the ground state at $\beta=-\infty$ of the directed polymer introduced above. Namely,

$$
\sup \left\{P\left(X_{i}=x_{i} \text { for } 1 \leq i \leq n\right): x_{i} \in \mathbb{Z}^{d}, \eta\left(i, x_{i}\right)=0\right\}=c_{1}^{n} \exp \left\{-c_{2} s_{p}^{-\alpha} T_{n}\left(\omega_{p}\right)\right\} .
$$

Furthermore, it is shown in Comets et al. (2015) that as $p \uparrow 1$, the ground state gives dominant contribution to the free energy:

$$
\varphi(p,-\infty) \sim-c_{2} \mu_{1}(1-p)^{-\alpha / d} .
$$

Remark 1.1. It is standard to show the existence of the free energy and the time constant by the subadditive ergodic theorem. However, it prevents us from getting more information about them, such as the continuity and the rate of convergence. In this paper, we will use a method of concentration of measures to get the rate of convergence in (1.2) and (1.5) and derive the continuity results as a corollary.

1.2. Main results. First, we shall present the results for our FPP. The first result is the concentration of the minimum passage time around the mean.

Theorem 1.2. For any $\delta>0$ there exist positive constants $C_{1}, C_{2}$, and $\lambda \in(0,1]$ which are independent of $p$ and $n$ such that for any $n \in \mathbb{N}$,

$$
Q\left(\left|T_{n}\left(\omega_{p}\right)-Q\left[T_{n}\left(\omega_{p}\right)\right]\right|>n^{\frac{1}{2}+\delta}\right)<C_{1} \exp \left\{-C_{2} n^{\lambda}\right\} .
$$

The next result is about the rate of convergence. It implies that the fluctuation exponent (see Krug and Spohn, 1991) is $\frac{1}{2}$ or less if exists.

Theorem 1.3. For any $\chi>1 / 2$, there exists positive constant $C_{1}$ which is independent of $p$ such that for any $n \in \mathbb{N}$,

$$
\left|n \mu_{p}-Q\left[T_{n}\left(\omega_{p}\right)\right]\right|<C_{1} n^{\chi}
$$


Combining (1.6) and (1.7), we have that for any $\chi>1 / 2$ there exist positive constants $C_{0}, C_{1}, C_{2}, \lambda$ which are independent of $p$ such that for any $n \in \mathbb{N}$,

$$
Q\left(\left|T_{n}\left(\omega_{p}\right)-n \mu_{p}\right|>2 C_{0} n^{\chi}\right)<C_{1} \exp \left\{-C_{2} n^{\lambda}\right\} .
$$

Corollary 1.4. $\mu_{p}$ is continuous in $p \in[0,1]$.

Corollary 1 is a slight extension of Theorem 1.5 in Comets et al. (2015) where the continuity is proved only at $p=1$.

Next, we move on to the results for the directed polymer model.

Theorem 1.5. For any $q \in[0,1), \delta>0$ and $\beta_{0} \in \mathbb{R}$, there exist positive constants $C_{1}, C_{2}$, and $\lambda \in(0,1)$ such that for any $p \in[0, q), \beta \in\left[-\infty, \beta_{0}\right]$ and $n \in \mathbb{N}$,

$$
Q\left(\left|\log Z_{n}^{\eta, \beta}-Q\left[\log Z_{n}^{\eta, \beta}\right]\right|>n^{\frac{1}{2}+\delta}\right)<C_{1} \exp \left\{-C_{2} n^{\lambda}\right\} .
$$

Theorem 1.6. For any $q \in[0,1), \chi>\frac{1}{2}$ and $\beta_{0} \in \mathbb{R}$, there exist positive constants $\epsilon, C_{0}-C_{2}$, and $\lambda \in(0,1)$ such that for any $p \in[0, q), \beta \in\left[-\infty, \beta_{0}\right]$ and $n \in \mathbb{N}$,

$$
\begin{aligned}
\left|n \varphi(p, \beta)-Q\left[\log Z_{n}^{\eta, \beta}\right]\right| & <C_{0} n^{\chi} \\
Q\left(\left|\log Z_{n}^{\eta, \beta}-n \varphi(p, \beta)\right|>2 C_{0} n^{\chi}\right) & <C_{1} \exp \left\{-C_{2} n^{\lambda}\right\} .
\end{aligned}
$$

The following theorem is an extension of Theorem 1.2 in Comets et al. (2015) where the continuity is proved only for $\alpha<d$ where $\alpha$ comes from (1.1).

Corollary 1.7. $\varphi(p, \beta)$ is jointly continuous on $[0,1) \times[-\infty, \infty)$.

Remark 1.8. Corollary 1.4 can in fact be proved by the "coupling method" in Comets et al. (2015). Nevertheless, we think the line of the argument-proving the continuity of a limiting quantity like the time constant via a concentration boundis of interest. In Corollary 1.7, the method of concentration indeed yields a better result than the rather bare-handed approach in Comets et al. (2015).

Remark 1.9. The method of this paper works for more general choices of (1.1). For example if $f(k)=c \exp \{-V(k)\}$ with $V$ satisfying $0<C_{1}<V^{\prime \prime}(x)<C_{2}$ for any $x$ with some positive constant $C_{1}$ and $C_{2}$ or $V^{\prime}$ being regularly varying with index $\beta>0$. Indeed, all of the statements including the key lemma below can be shown by the essentially the same way for these choices.

1.3. Organization of the paper. The rest of the paper is organized as follows. In Section 2, we start with the proof of Theorems 1.2. We divide the proof into two parts; $\alpha \leq 1$ or $\alpha>1$. We first prove the case $\alpha \leq 1$ which is relatively easy. To prove the other case, we need a key lemma in Section 2.2 which gives a control on the maximum jump of optimal paths as a special case. This lemma is the essential part of this paper. By using the same lemma, polymers with large jumps can be shown to have negligible weights under the Gibbs measure. By restricting our attention to the polymers with small jumps, we can check the condition for the entropy method (Theorem 6.7 in Boucheron et al., 2013) to show the concentration bound and obtain Theorem 1.5. In Section 3, we discuss the non-random fluctuations and prove Theorems 1.3 and 1.6 by using Theorems 1.2 and 1.5 , respectively. The proofs are based on the argument in Zhang (2010). Finally, we prove Corollaries 1.4 and 1.7 in Section 4 as a consequence of the results of Section 3. 


\section{Proof of the concentration around the mean}

For any $n$-path $\gamma \in\left(\mathbb{R}^{d}\right)^{\{1, \cdots, n\}}$, we write $\gamma(k)$ for the $k$-th point of $\gamma$, and $\Delta \gamma(k):=|\gamma(k)-\gamma(k-1)|_{1}$ with the convention $\gamma(0)=0$.

Definition 2.1. For any $n$-path $\gamma \in\left(\mathbb{R}^{d}\right)^{\{1, \cdots, n\}}$, the passage time $t(\gamma)$ is defined by

$$
t(\gamma)=\sum_{i=1}^{n} \Delta \gamma(i)^{\alpha}
$$

2.1. Concentration for the FPP with $0<\alpha \leq 1$. We first calculate the probability that $\omega_{p}$ has no points inside a box of size $r>0$.

Lemma 2.2. There exist $C_{1}, C_{2}>0$ independent of $p$ such that for any sufficiently large $r>0$,

$$
Q\left(\omega_{p} \cap[0, r]^{d}=\emptyset\right) \leq C_{1} \exp \left(-C_{2} r^{d}\right) .
$$

Proof: Note that for sufficiently large $r>0,[0, r]^{d} \cap s_{p} \mathbb{Z}^{d}$ has at least $\left(2^{-1} s_{p}^{-1} r\right)^{d}$ points, where $s_{p}=(1-p)^{1 / d}$. Thus

$$
Q\left(\omega_{p} \cap[0, r]^{d}=\emptyset\right) \leq\left(p^{(1-p)^{-1}}\right)^{2^{-1} r^{d}} .
$$

Since it is easy to check that $\sup _{p \in[0,1]} p^{(1-p)^{-1}}<1$, we complete the proof.

Proof of (1.6) for $0<\alpha \leq 1$ : We prove (1.6) by using a martingale difference method. We introduce the filtration

$$
\mathcal{G}_{m}=\sigma\left(\left.\omega_{p}\right|_{[1, m] \times \mathbb{R}^{d}}\right)
$$

and decompose the deviation from the mean into the sum of martingale differences as

$$
\begin{aligned}
& T_{n}\left(\omega_{p}\right)-Q\left[T_{n}\left(\omega_{p}\right)\right] \\
& =\sum_{m=1}^{n}\left(Q\left[T_{n}\left(\omega_{p}\right) \mid \mathcal{G}_{m}\right]-Q\left[T_{n}\left(\omega_{p}\right) \mid \mathcal{G}_{m-1}\right]\right) \\
& =: \sum_{m=1}^{n} \Delta_{m} .
\end{aligned}
$$

We are going to prove that there exists non-random constant $c>0$ independent of $p$ such that

$$
Q\left[\exp \left\{c\left|\Delta_{m}\right|^{d / \alpha}\right\} \mid \mathcal{G}_{m-1}\right] \leq c^{-1}
$$

$Q$-almost surely. Then (1.6) follows by a concentration inequality for martingales, for example, Theorem 1.1 in Liu and Watbled (2009). Before proving it, let us introduce some notation. Given two configurations $\omega_{p}$ and $\omega_{p}^{\prime}$ and $m \in \mathbb{N}$, we define the new configuration $\left[\omega_{p}, \omega_{p}^{\prime}\right]_{m}$ by

$$
\left.\omega_{p}\right|_{[1, m] \times \mathbb{R}^{d}}+\left.\omega_{p}^{\prime}\right|_{[m+1, \infty) \times \mathbb{R}^{d}} .
$$

Let $\pi_{n}^{(m)}=\left(i, \pi_{n}^{(m)}(i)\right)_{i=0}^{n}$ be a minimizing path for this configuration chosen by a deterministic algorithm (if not unique). Given $(n, x),(m, y) \in \mathbb{N} \times \mathbb{R}^{d}$, we define $|(n, x)-(m, y)|_{1}=|x-y|_{1}$. For a time-space point $(k, x) \in \mathbb{N} \times \mathbb{R}^{d}$, we define $(k, x)^{* \omega_{p}}$ to be a point in $\left.\omega_{p}\right|_{\{k\} \times \mathbb{R}^{d}}$ closest to $(k, x)$ with a deterministic rule to break ties and sometimes use the convention that $(k, x)^{* \omega_{p}}=\left(k, x^{* \omega_{p}}\right)$ with a slight 
abuse of notation.

It is easy to check that the law of $(k, x)^{* \omega_{p}}$ is independent of $(k, x)$ and by Lemma 2.2, there exist $C_{1}, C_{2}>0$ independent of $p$ such that for any sufficiently large $r>0$,

$$
Q\left(\left|(k, x)-(k, x)^{* \omega_{p}}\right|_{1}>r\right) \leq C_{1} \exp \left(-C_{2} r^{d}\right) .
$$

Now we rewrite the martingale difference as

$$
\Delta_{m}=\int Q\left(\mathrm{~d} \omega_{p}^{\prime}\right)\left(T_{n}\left(\left[\omega_{p}, \omega_{p}^{\prime}\right]_{m}\right)-T_{n}\left(\left[\omega_{p}, \omega_{p}^{\prime}\right]_{m-1}\right)\right) .
$$

We can bound $T_{n}\left(\left[\omega_{p}, \omega_{p}^{\prime}\right]_{m-1}\right)$ from above by the passage time of the path

$$
\pi_{n}^{(m)}(0), \ldots, \pi_{n}^{(m)}(m-1), \pi_{n}^{(m)}(m)^{* \omega_{p}^{\prime}}, \pi_{n}^{(m)}(m+1), \ldots, \pi_{n}^{(m)}(n) .
$$

By $\alpha \leq 1$ we have

$$
\begin{aligned}
& \left|\pi_{n}^{(m)}(m \pm 1)-\pi_{n}^{(m)}(m)^{* \omega_{p}^{\prime}}\right|_{1}^{\alpha} \\
& \quad \leq\left|\pi_{n}^{(m)}(m \pm 1)-\pi_{n}^{(m)}(m)\right|_{1}^{\alpha}+\left|\pi_{n}^{(m)}(m)-\pi_{n}^{(m)}(m)^{* \omega_{p}^{\prime}}\right|_{1}^{\alpha} .
\end{aligned}
$$

These lead to

$$
T_{n}\left(\left[\omega_{p}, \omega_{p}^{\prime}\right]_{m}\right)-T_{n}\left(\left[\omega_{p}, \omega_{p}^{\prime}\right]_{m-1}\right) \geq-2\left|\pi_{n}^{(m)}(m)-\pi_{n}^{(m)}(m)^{* \omega_{p}^{\prime}}\right|_{1}^{\alpha},
$$

and we get

$$
\Delta_{m} \geq-2 \int Q\left(\mathrm{~d} \omega_{p}^{\prime}\right)\left|\pi_{n}^{(m)}(m)-\pi_{n}^{(m)}(m)^{* \omega_{p}^{\prime}}\right|_{1}^{\alpha} .
$$

Similarly, we have

$$
\Delta_{m} \leq 2 \int Q\left(\mathrm{~d} \omega_{p}^{\prime}\right)\left|\pi_{n}^{(m-1)}(m)-\pi_{n}^{(m-1)}(m)^{* \omega_{p}}\right|_{1}^{\alpha} .
$$

Note that the laws of right hand side of (2.6) and (2.7) are independent of $\mathcal{G}_{m-1}$. By Jensen's inequality, (2.6) and (2.7), if we take $c$ sufficiently small depending only on $C_{1}, C_{2}>0$ of $(2.3)$, we have

$$
\begin{aligned}
& Q\left[\exp \left\{c\left|\Delta_{m}\right|^{d / \alpha}\right\} \mid \mathcal{G}_{m-1}\right] \\
\leq & \int Q\left(d \omega_{p}\right) \int Q\left(d \omega_{p}^{\prime}\right) \exp \left(2 c\left|\pi_{n}^{(m)}(m)-\pi_{n}^{(m)}(m)^{* \omega_{p}^{\prime}}\right|_{1}^{d}\right) \\
& \quad+\int Q\left(d \omega_{p}\right) \int Q\left(d \omega_{p}^{\prime}\right) \exp \left(2 c\left|\pi_{n}^{(m-1)}(m)-\pi_{n}^{(m-1)}(m)^{* \omega_{p}}\right|_{1}^{d}\right) \\
= & 2 \int Q\left(d \omega_{p}\right) \exp \left(2 c \mid(k, x)-(k, x)^{\left.\left.* \omega_{p}\right|_{1} ^{d}\right) \leq c^{-1} .}\right.
\end{aligned}
$$

2.2. Key lemma (The uniform bound for jumps). As mentioned above, it is more difficult to prove Theorem 1.2 in the case $\alpha>1$. Indeed, we need to estimate the change of minimum passage time when we replace the configuration on a section 
by another one, but we do not have the triangular inequality (2.5). A natural alternative way is to use Taylor's theorem as follows:

$$
\begin{aligned}
& \left|T_{n}\left(\left[\omega_{p}, \omega_{p}^{\prime}\right]_{m}\right)-T_{n}\left(\left[\omega_{p}, \omega_{p}^{\prime}\right]_{m-1}\right)\right| \\
& \leq C\left(\left|\pi_{n}^{(m)}(m+1)-\pi_{n}^{(m)}(m)\right|_{1}^{\alpha-1}\left|\pi_{n}^{(m)}(m)-\pi_{n}^{(m)}(m)^{* \omega_{p}^{\prime}}\right|_{1}\right. \\
& \left.\quad+\left|\pi_{n}^{(m)}(m)-\pi_{n}^{(m)}(m-1)\right|_{1}^{\alpha-1}\left|\pi_{n}^{(m)}(m)-\pi_{n}^{(m)}(m)^{* \omega_{p}^{\prime}}\right|_{1}\right),
\end{aligned}
$$

with some positive constant $C$. Then, the jump size of the optimal path appears in this change and we need to show that it is not too large. We shall state it in a slightly generalized way that is useful in the study of the directed polymer model.

Given a configuration $\omega$ and $n$-path $\gamma$, we write $\gamma \subset \omega$ if $(i, \gamma(i)) \in \omega$ for any $i=1, \cdots, n$. We say that $\omega \subset \mathbb{N} \times \mathbb{R}^{d}$ has $\theta$-property if

$$
\omega \cap\left(i, x+\left[0, n^{\theta}\right)^{d}\right) \neq \emptyset \text { for any } i \in \mathbb{N}, x \in \mathbb{Z}^{d} .
$$

Intuitively, $\theta$-property means that configration has no big vacant regions. As we shall see in Lemma 2.4 that the $\theta$-property scarcely influence on the minimum passage time. The following lemma plays a key role throughout all the results in this paper. Roughly speaking, any polymer has no big jumps or we can find another polymer with a smaller passage time.

Lemma 2.3. Suppose that $\alpha>1$. For any $\zeta>0$, there exist $\theta>0$ and $N=$ $N(\theta) \in \mathbb{N}$ such that if $n>N, s \in\{1, \cdots, n\}$ and a configuration $\omega^{\prime} \subset \mathbb{N} \times \mathbb{R}^{d}$ satisfies $\theta$-property, then for any n-path $\gamma$, one of the following holds:

(i) $\max \{\Delta \gamma(s), \Delta \gamma(s+1)\} \leq n^{\zeta}$ with the convention $\Delta \gamma(n+1)=0$,

(ii) There exist an $n$-path $\gamma^{\prime}$ and $h \in \mathbb{N}$ such that $\gamma(i)=\gamma^{\prime}(i)$ for any $i \notin$ $[s, s+h-1],\left(i, \gamma^{\prime}(i)\right) \in \omega^{\prime}$ for $i \in[s, s+h-1]$ and

$$
t\left(\gamma^{\prime}\right)+(h+1) n^{\theta} \leq t(\gamma) .
$$

The proof of Lemma 1 is not long but a bit complicated. Let us explain the idea of the proof in the case where $\gamma$ is the minimizing path for $\omega^{\prime}$. Let $A_{0}$ be the point where next jump is larger than $n^{\zeta}$ and introduce a sequence of large numbers satisfying $n^{\zeta} \gg \mathcal{L}_{1} \gg \mathcal{L}_{2} \gg \cdots$.

We draw cones $C_{k}$ with slope $\mathcal{L}_{k}$ starting at $A_{0}$ and let $A_{k}$ be the first point where the optimal path $\gamma$ touches it. Then we draw a straight line between $A_{0}$ and $A_{k}$. Thanks to the $\theta$-property, we can find a path $\gamma_{k}^{\prime} \subset \bar{\omega}$ close to this straight line. Due to the convexity of $|\cdot|_{1}^{\alpha}$, average jumps are better (See Figure 2.1). This yields that, if we forget about the restriction $\subset \bar{\omega}$, the straight line connecting $A_{0}$ and $A_{k}$ is the optimal path when $\alpha>1$. On the other hand, by definition, $\gamma$ has a smaller passage time than that of $\gamma_{k}^{\prime}$. Then it is natural to expect that most of the jumps of $\gamma$ between $A_{0}$ and $A_{k}$ are close to $\mathcal{L}_{k}$ in size. However in the first $A_{0} \rightarrow A_{k-1}$ segment, most of the jumps are close to $\mathcal{L}_{k-1}$ for the same reason and hence far from $\mathcal{L}_{k}$. This implies that the duration of $A_{0} \rightarrow A_{k}$ is much longer than that of $A_{0} \rightarrow A_{k-1}$. Based on this observation, we can prove that $A_{k}$ goes beyond the $n$-th section before $\mathcal{L}_{k}$ becomes small. This implies that the optimal path $\gamma$ stays outside a cone of very large angle until $n$. This is very unlikely because the optimal path has too much passage time between $A_{0}$ and $n$-th section, and one can indeed derive a contradiction. 


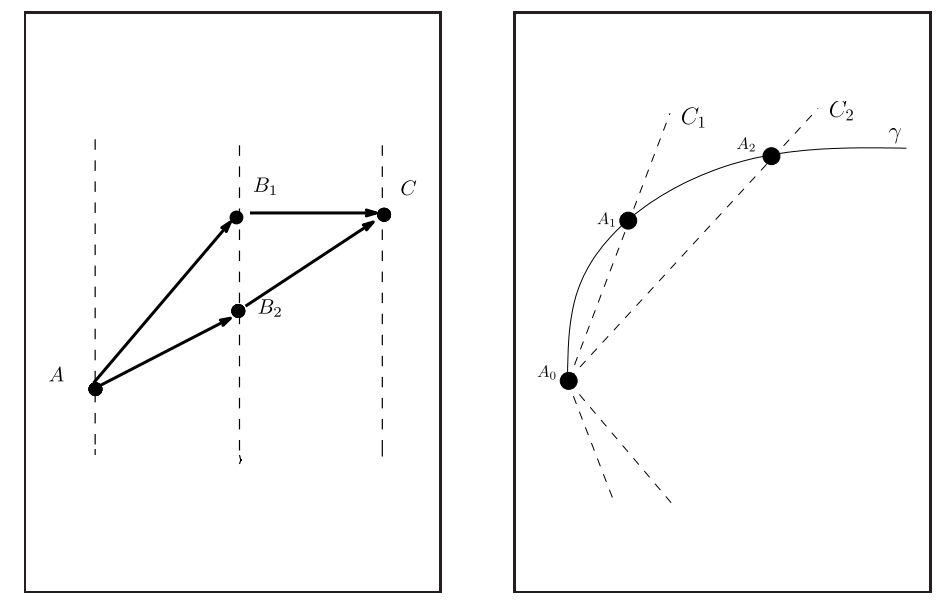

FiguRE 2.1.

Left: The passage time of $A B_{2} C$ is smaller than that of $A B_{1} C$ when $\alpha>1$.

Right: Drawing cones, we investigate $\gamma$ between $A_{0}$ and $A_{k}$ inductively.

Proof: Let $m \in \mathbb{N}$ such that $m>5 / \zeta$, and we take $\theta>0$ sufficiently small and $N$ sufficiently large such that

$$
2^{5} m \alpha \theta<\zeta \text { and } N^{\min \{\alpha-1,1\} \theta}>2^{\alpha+4} \alpha^{2}(\alpha-1)^{-1}+\left(2^{\frac{1}{\alpha}}-1\right)^{-1} .
$$

Suppose that neither (i) nor (ii) holds and we shall derive a contradiction. This means that we suppose the following two conditions:

(i') $\max \{\Delta \gamma(s), \Delta \gamma(s+1)\}>n^{\zeta}$,

(ii') For any $n$-path $\gamma^{\prime}$ and $h>0$ such that $\gamma(i)=\gamma^{\prime}(i)$ for $i \notin[s, s+h-1]$, and $\left(i, \gamma^{\prime}(i)\right) \in \omega^{\prime}$ for $i \in[s, s+h], t\left(\gamma^{\prime}\right)+(h+1) n^{\theta}>t(\gamma)$.

We only treat the case $\Delta \gamma(s)>n^{\zeta}$ since the other case $\Delta \gamma(s+1)>\Delta \gamma(s)$ can be dealt with by simply replacing $s$ by $s+1$ in the following proof.

$\underline{\text { Step } 1}$ (Preliminary bounds): For any $k \in\{1, \cdots, m\}$, let

$$
\ell_{k}:=\inf \left\{\ell \in[s, n] \cap \mathbb{N}: \frac{|\gamma(\ell)-\gamma(s-1)|_{1}}{\ell-s+1} \leq n^{\zeta-(2 k-1) \theta}\right\} .
$$

The points $(s-1, \gamma(s-1))$ and $\left(\ell_{k}, \gamma\left(\ell_{k}\right)\right)$ correspond to $A_{0}$ and $A_{k}$ in Figure 2.1, respectively. By the $\theta$-property of $\omega^{\prime}$, there exists an $n$-path $\gamma^{\prime}$ such that $\gamma(i)=\gamma^{\prime}(i)$ for any $i \leq s-1$ and $\left(i, \gamma^{\prime}(i)\right) \in \omega^{\prime}, \Delta \gamma^{\prime}(i) \leq d n^{\theta}$ for any $i \geq s$. If the range of the infimum of (2.8) is empty, then

$$
t(\gamma)-t\left(\gamma^{\prime}\right)-(n-s+1) n^{\theta} \geq(n-s+1)\left(n^{\zeta-(2 k-1) \theta}-d n^{\theta}-n^{\theta}\right) \geq 0,
$$

and it contradicts (ii'). Hence, the range is not void for $k \leq m$ and $\ell_{k} \leq n$.

Let $\bar{\gamma}_{k}$ be a straight line drawn between $(s-1, \gamma(s-1))$ and $\left(\ell_{k}, \gamma\left(\ell_{k}\right)\right)$. We write $\mathcal{L}_{k}$ for a slope $\frac{\left|\gamma\left(\ell_{k}\right)-\gamma(s-1)\right|_{1}}{\ell_{k}-s+1}$ of $\bar{\gamma}_{k}$. Since $\omega^{\prime}$ has $\theta$-property, there exists an $n$-path $\gamma_{k}^{\prime}$ such that $\left(j, \gamma_{k}^{\prime}(j)\right) \in \omega^{\prime},\left|\bar{\gamma}_{k}(j)-\gamma_{k}^{\prime}(j)\right|_{1} \leq d n^{\theta}$ for all $s \leq j<\ell_{k}$, and $\gamma_{k}^{\prime}(\ell)=\gamma(\ell)$ for $\ell \leq s-1$ or $\ell \geq \ell_{k}$. Set $R_{k}:=\ell_{k}-s, D_{k}:=\left\{i \in\left\{0, \cdots, R_{k}\right\}\right.$ : $\left.\Delta \gamma(s+i) \geq n^{\zeta-2 k \theta}\right\}, D_{k}^{c}:=\left\{0, \cdots, R_{k}\right\} \backslash D_{k}$. The set $D_{k}$ corresponds to the time 
when $\Delta \gamma$ is close to $\mathcal{L}_{k}$, though we put one-sided inequality in the definition.

Let $\delta_{j, k}:=\Delta \gamma(s+j)-\mathcal{L}_{k}$ and $\delta_{j, k}^{\prime}:=\Delta \gamma_{k}^{\prime}(s+j)-\mathcal{L}_{k}$. We note that for any $k \geq 1,\left|\delta_{j, k}^{\prime}\right| \leq 2 d n^{\theta}$. Moreover since $\gamma_{k}$ and $\gamma_{k}^{\prime}$ start and end at the same points, we have

$$
\sum_{i=0}^{R_{k}} \delta_{i, k} \geq 0, \quad \sum_{i=0}^{R_{k}} \delta_{i, k}^{\prime} \geq 0 .
$$

Note that by convexity of $|\cdot|_{1}^{\alpha}$,

$$
\left(\mathcal{L}_{k}+\delta_{i, k}\right)^{\alpha}-\mathcal{L}_{k}^{\alpha}-\alpha \mathcal{L}_{k}^{\alpha-1} \delta_{i, k} \geq 0 .
$$

These positivities (2.8) and (2.9) will be crucial in the proof.

Step $2\left(D_{k+1}\right.$ is much larger than $\left.D_{k}\right)$ : We have

$$
n^{\zeta-(2 k-1) \theta}-n^{\zeta-2 k \theta} \leq \mathcal{L}_{k} \leq n^{\zeta-(2 k-1) \theta} .
$$

The proof is deferred to the end of this subsection.

We will show that $\# D_{k+1} \geq n^{\zeta / 2} \# D_{k}$ for any $k<m$. Once we get this, by iteration and $D_{0} \geq 1, \# D_{m} \geq n^{\zeta m / 2} \geq n^{5 / 4}$ holds, which is a contradiction. Thus the lemma follows.

Use (2.8) to obtain that

$$
\begin{aligned}
& \sum_{i=0}^{R_{k+1}}\left\{\left(\mathcal{L}_{k+1}+\delta_{i, k+1}\right)^{\alpha}-\mathcal{L}_{k+1}^{\alpha}-\alpha \mathcal{L}_{k+1}^{\alpha-1} \delta_{i, k+1}\right\} \\
= & \sum_{i=0}^{R_{k+1}}\left\{\Delta \gamma(i+s)^{\alpha}-\mathcal{L}_{k+1}^{\alpha}\right\}-\alpha \mathcal{L}_{k+1}^{\alpha-1} \sum_{i=0}^{R_{k+1}} \delta_{i, k+1} \\
\leq & \sum_{i=0}^{R_{k+1}}\left\{\Delta \gamma(i+s)^{\alpha}-\mathcal{L}_{k+1}^{\alpha}\right\} .
\end{aligned}
$$

When $i \in D_{k+1}^{c}$, by $(2.10)$ we have $-\delta_{i, k+1} \geq \mathcal{L}_{k+1}-n^{\zeta-2(k+1) \theta} \geq \frac{\alpha+1}{2 \alpha} \mathcal{L}_{k+1}$ for sufficiently large $n$ and hence

$$
\left(\mathcal{L}_{k+1}+\delta_{i, k+1}\right)^{\alpha}-\mathcal{L}_{k+1}^{\alpha}-\alpha \mathcal{L}_{k+1}^{\alpha-1} \delta_{i, k+1} \geq \frac{\alpha-1}{2} \mathcal{L}_{k+1}^{\alpha} .
$$

This together with (2.9) and $D_{k} \subset D_{k+1}$ shows that the left hand side of (2.11) is further bounded from below by

$$
\begin{gathered}
\sum_{i \in D_{k} \cup D_{k+1}^{c}}\left\{\left(\mathcal{L}_{k+1}+\delta_{i, k+1}\right)^{\alpha}-\mathcal{L}_{k+1}^{\alpha}-\alpha \mathcal{L}_{k+1}^{\alpha-1} \delta_{i, k+1}\right\} \\
\geq \sum_{i \in D_{k}}\left\{\left(\mathcal{L}_{k+1}+\delta_{i, k+1}\right)^{\alpha}-\mathcal{L}_{k+1}^{\alpha}-\alpha \mathcal{L}_{k+1}^{\alpha-1} \delta_{i, k+1}\right\}+\frac{\alpha-1}{2} \sum_{i \in D_{k+1}^{c}} \mathcal{L}_{k+1}^{\alpha} .
\end{gathered}
$$

On the other hand, due to (ii'), the right hand side of (2.11) is bounded from above by

$$
\sum_{i=0}^{R_{k+1}}\left\{\left(\mathcal{L}_{k+1}+\delta_{i, k+1}^{\prime}\right)^{\alpha}-\mathcal{L}_{k+1}^{\alpha}+n^{\theta}\right\} .
$$


The fact that $|a+b|^{\alpha}-|a|^{\alpha} \leq \alpha|b-a|(|a|+|b|)^{\alpha-1}$ for $a, b \in \mathbb{R}$ together with (2.10) and $\left|\delta_{i, k+1}^{\prime}\right| \leq 2 d n^{\theta}$ leads to that for any $i \in\left\{0, \cdots, R_{k+1}\right\}$,

$$
\left(\mathcal{L}_{k+1}+\delta_{i, k+1}^{\prime}\right)^{\alpha}-\mathcal{L}_{k+1}^{\alpha}+n^{\theta} \leq 4 d \alpha n^{(\alpha-1)(\zeta-(2 k+1) \theta)+\theta} \leq \frac{\alpha-1}{2} \mathcal{L}_{k+1}^{\alpha} .
$$

Thus, (2.13) is further bounded from above by

$$
\begin{aligned}
& \sum_{i \in D_{k+1}}\left\{\left(\mathcal{L}_{k+1}+\delta_{i, k+1}^{\prime}\right)^{\alpha}-\mathcal{L}_{k+1}^{\alpha}+n^{\theta}\right\}+\sum_{i \in D_{k+1}^{c}}\left\{\left(\mathcal{L}_{k+1}+\delta_{i, k+1}^{\prime}\right)^{\alpha}-\mathcal{L}_{k+1}^{\alpha}+n^{\theta}\right\} \\
\leq & \sum_{i \in D_{k+1}} 4 d \alpha n^{(\alpha-1)(\zeta-(2 k+1) \theta)+\theta}+\frac{\alpha-1}{2} \sum_{i \in D_{k+1}^{c}} \mathcal{L}_{k+1}^{\alpha} .
\end{aligned}
$$

With these observations, since $\delta_{i, k+1}$ is much larger than $\mathcal{L}_{k+1}$ for $i \in D_{k}$, we have

$$
\begin{aligned}
& 4 d \alpha \# D_{k+1} n^{(\alpha-1)(\zeta-(2 k+1) \theta)+\theta} \\
\geq & \sum_{i \in D_{k}}\left\{\left(\mathcal{L}_{k+1}+\delta_{i, k+1}\right)^{\alpha}-\mathcal{L}_{k+1}^{\alpha}-\alpha \mathcal{L}_{k+1}^{\alpha-1} \delta_{i, k+1}\right\} \\
\geq & \# D_{k} \min _{i \in D_{k}}\left\{\left(\mathcal{L}_{k+1}+\delta_{i, k+1}\right)^{\alpha}-\mathcal{L}_{k+1}^{\alpha}-\alpha \mathcal{L}_{k+1}^{\alpha-1} \delta_{i, k+1}\right\} \\
\geq & \frac{1}{2} \# D_{k} n^{\alpha(\zeta-2 k \theta)} .
\end{aligned}
$$

Rearranging it, we obtain $\# D_{k+1} \geq n^{\zeta / 2} \# D_{k}$ as desired.

Proof of (2.10): Our strategy is to show that the last jump $\Delta \gamma\left(s+R_{k}\right)$ is small or $R_{k}$ is large. We choose $\zeta^{\prime}$ such that $n^{\zeta^{\prime}}=\Delta \gamma\left(s+R_{k}\right)$. By (ii') and (2.8),

$$
\sum_{i=0}^{R_{k}}\left\{\left(\mathcal{L}_{k}+\delta_{i, k}^{\prime}\right)^{\alpha}-\mathcal{L}_{k}^{\alpha}+n^{\theta}\right\} \geq \sum_{i=0}^{R_{k}}\left\{\left(\mathcal{L}_{k}+\delta_{i, k}\right)^{\alpha}-\mathcal{L}_{k}^{\alpha}-\alpha \mathcal{L}_{k}^{\alpha-1} \delta_{i, k}\right\} .
$$

By arguments similar to (2.14), we get $\left(\mathcal{L}_{k}+\delta_{i, k}^{\prime}\right)^{\alpha}-\mathcal{L}_{k}^{\alpha} \leq 4 d \alpha \mathcal{L}_{k}^{\alpha-1} n^{\theta}$. Thus, the left hand side of (2.16) is bounded from above by

$$
\left(R_{k}+1\right)\left(4 d \alpha \mathcal{L}_{k}^{\alpha-1} n^{\theta}+n^{\theta}\right) \leq R_{k} n^{\zeta(\alpha-1)+2 \theta} .
$$

On the other hand, by (2.9) and $\delta_{0, k}=\Delta \gamma(s)-\mathcal{L}_{k} \geq n^{\zeta} / 2 \gg n^{\zeta-(2 k-1) \theta} \geq \mathcal{L}_{k}$, the right hand side of (2.16) is bounded from below by

$$
\begin{aligned}
& \left(\left(\mathcal{L}_{k}+\delta_{0, k}\right)^{\alpha}-\mathcal{L}_{k}^{\alpha}-\alpha \mathcal{L}_{k}^{\alpha-1} \delta_{0, k}\right)+\left(\left(\mathcal{L}_{k}+\delta_{R_{k}, k}\right)^{\alpha}-\mathcal{L}_{k}^{\alpha}-\alpha \mathcal{L}_{k}^{\alpha-1} \delta_{R_{k}, k}\right) \\
\geq & \frac{1}{2} \max \left\{n^{\zeta \alpha}, n^{\zeta^{\prime} \alpha}\right\} .
\end{aligned}
$$

Consequently, we have

$$
R_{k} \geq \frac{1}{2} \max \left\{n^{\zeta-2 \theta}, n^{\zeta^{\prime} \alpha-(\alpha-1) \zeta-2 \theta}\right\} \geq \max \left\{n^{\zeta-3 \theta}, n^{\zeta^{\prime}-3 \theta}\right\} .
$$


By the definition of $R_{k}$, we have $\left|\gamma\left(s+R_{k}-1\right)-\gamma(s-1)\right|_{1} \geq R_{k} n^{\zeta-(2 k-1) \theta}$. This yields

$$
\begin{aligned}
\mathcal{L}_{k} & \geq \frac{1}{1+R_{k}}\left(\left|\gamma\left(s+R_{k}-1\right)-\gamma(s-1)\right|_{1}-\Delta \gamma\left(s+R_{k}\right)\right) \\
& \geq n^{\zeta-(2 k-1) \theta}\left(1-\frac{1}{1+R_{k}}\right)-n^{3 \theta} \\
& \geq n^{\zeta-(2 k-1) \theta}-n^{\zeta-2 k \theta} .
\end{aligned}
$$

We have $\mathcal{L}_{k} \leq n^{\zeta-(2 k-1) \theta}$ by the construction and (2.10) follows.

2.3. Concentration for the FPP with $\alpha>1$. In this subsection, we prove Theorem 1.2 for $\alpha>1$. We fix a small $\theta>0$. Given a configuration $\omega_{p} \subset \mathbb{N} \times \mathbb{R}^{d}$, we define a new configuration $\bar{\omega}_{p}$ as

$$
\bar{\omega}_{p}=\omega_{p}+\sum_{(k, x) \in \mathbb{N} \times n^{\theta} \mathbb{Z}^{d}} 1_{\left\{\omega\left(\{k\} \times\left(x+\left[0, n^{\theta}\right)^{d}\right)\right)=0\right\}} \delta_{(k, x)},
$$

i.e. when we find a large vacant box, we artificially add an $\omega$-point at a corner. We first recall Lemma 3.3 in Comets et al. (2015) which shows that $T_{n}\left(\omega_{p}\right)$ and $T_{n}\left(\bar{\omega}_{p}\right)$ are essentially the same.

Lemma 2.4. There exists $c>0$ independent of $p$ such that for sufficiently large $n \in \mathbb{N}$,

$$
\begin{aligned}
\max \left\{Q \left(T_{n}\left(\omega_{p}\right) \neq\right.\right. & \left.\left.T_{n}\left(\bar{\omega}_{p}\right)\right), Q\left[\left|T_{n}\left(\omega_{p}\right)-T_{n}\left(\bar{\omega}_{p}\right)\right|\right]\right\} \\
& \leq \exp \left\{-c n^{d \theta}\right\} .
\end{aligned}
$$

Proof: This is proved only for $\omega_{1}$ in Comets et al. (2015) but the same proof works for general $\omega_{p}$. In fact, it is proved that the probability that the set of all optimal paths for $\omega_{p}$ coincides with that for $\bar{\omega}_{p}$ is larger than $1-\exp \left(-c n^{d \theta}\right)$. The proof of Lemma 2.9 below contains a very similar argument.

Proof of (1.6) for $\alpha>1$ : Fix $\delta>0$. Let us denote by $\bar{\omega}_{p}^{(m)}$ the point process obtained by replacing its $\{m\} \times \mathbb{R}^{d}$-section by another configuration $\bar{\omega}_{p}^{\prime}$. We are going to use the so-called entropy method and it requires a bound on

$$
\sum_{m=1}^{n}\left(\sup _{\omega_{p}^{\prime}}\left|T_{n}\left(\bar{\omega}_{p}^{(m)}\right)-T_{n}\left(\bar{\omega}_{p}\right)\right|\right)^{2},
$$

where the supremum is taken over all configrations $\omega_{p}^{\prime}$.

Lemma 2.5. Suppose that $\alpha>1$. For any $\zeta>0$, there exist $\theta \in(0, \zeta)$ and $N=N(\theta) \in \mathbb{N}$ such that for any $n>N, 1 \leq i \leq n, \omega_{p} \subset \mathbb{N} \times \mathbb{R}^{d}$, and a minimizing path $\pi_{n}$ for $\bar{\omega}_{p}$,

$$
\Delta \pi_{n}(i) \leq n^{\zeta}
$$

Proof: Let $\omega^{\prime}=\bar{\omega}_{p}$ and $\gamma=\pi_{n}$ in Lemma 2.3. Then for any $s \in\{1, \cdots, n\}$, (i) or (ii) holds. If (ii) holds, it contradicts that $\pi_{n}$ is a minimizing path. It follows that (i) holds and we get the desired conclusion.

By using Lemma 2.5, we can bound the summands of (2.18) from above by $n^{o(1)}$ and consequently (2.18) itself by $n^{1+o(1)}$. 
Lemma 2.6. For any $\zeta>0$, there exist $\theta, N>0$ such that for all $n \geq N$ and $\omega_{p} \subset \mathbb{N} \times \mathbb{R}^{d}$,

$$
\left|\Delta_{m}\right| \leq 4 n^{\zeta \alpha},
$$

where $\Delta_{m}:=\sup _{\omega^{\prime}}\left|T_{n}\left(\bar{\omega}_{p}^{(m)}\right)-T_{n}\left(\bar{\omega}_{p}\right)\right|$.

Proof: We prove the lower bound for $\Delta_{m}$. The upper bound can be proved similarly. Fix some $\omega_{p}$ and $\omega_{p}^{\prime}$. Let $\pi_{n}$ denote a minimizing path for $T_{n}\left(\bar{\omega}_{p}\right)$, chosen by a deterministic algorithm if not unique. We define a new point $\tilde{\pi}_{n}^{(m)}(m)$ as a point in $\left.\bar{\omega}_{p}^{\prime}\right|_{\{m\} \times \mathbb{R}^{d}}$ satisfying

$$
\left|\pi_{n}(m)-\tilde{\pi}_{n}^{(m)}(m)\right|_{1} \leq d n^{\theta} .
$$

Then we can bound $T_{n}\left(\bar{\omega}_{p}^{(m)}\right)$ from above by the passage time of the path

$$
\tilde{\pi}_{n}^{(m)}=\left(\pi_{n}(1), \ldots, \pi_{n}(m-1), \tilde{\pi}_{n}^{(m)}(m), \pi_{n}(m+1), \ldots, \pi_{n}(n)\right) .
$$

By using Lemma 2.5 together with (2.19), for sufficiently small $\theta$ and large $n$, we get

$$
\begin{aligned}
& T_{n}\left(\bar{\omega}_{p}^{(m)}\right)-T_{n}\left(\bar{\omega}_{p}\right) \leq t\left(\tilde{\pi}_{n}^{(m)}\right)-t\left(\pi_{n}\right) \\
\leq & \left|\tilde{\pi}_{n}^{(m)}(m-1)-\tilde{\pi}_{n}^{(m)}(m)\right|_{1}^{\alpha}+\left|\tilde{\pi}_{n}^{(m)}(m)-\tilde{\pi}_{n}^{(m)}(m+1)\right|_{1}^{\alpha} \\
\leq & 2\left(n^{\zeta}+d n^{\theta}\right)^{\alpha}<4 n^{\zeta \alpha}
\end{aligned}
$$

as desired. The reverse inequality can be proved by a similar way.

If we take $\zeta$ sufficiently small, (2.18) is less than $C n^{1+\delta}$. Then, Theorem 6.7 in Boucheron et al. (2013) yields that there exists $c>0$ independent of $p$ such that

$$
Q\left(\left|T_{n}\left(\bar{\omega}_{p}\right)-Q\left[T_{n}\left(\bar{\omega}_{p}\right)\right]\right|>n^{\frac{1}{2}+\delta}\right) \leq \exp \left\{-c n^{1-\delta}\right\} .
$$

By Lemma 2.4, there exists $c^{\prime}>0$ independent of $p$ such that

$$
\begin{aligned}
& Q\left(\left|T_{n}\left(\omega_{p}\right)-Q\left[T_{n}\left(\omega_{p}\right)\right]\right|>2 n^{\frac{1}{2}+\delta}\right) \\
& \leq Q\left(\left|T_{n}\left(\bar{\omega}_{p}\right)-Q\left[T_{n}\left(\bar{\omega}_{p}\right)\right]\right|>n^{\frac{1}{2}+\delta}\right)+Q\left(T_{n}\left(\omega_{p}\right) \neq T_{n}\left(\bar{\omega}_{p}\right)\right) \\
& \leq \exp \left\{-c^{\prime} n^{1-\delta}\right\}+\exp \left\{-c^{\prime} n^{d \theta}\right\},
\end{aligned}
$$

which implies (1.6).

By the proof of Lemma 2.4, Lemma 2.6 and the Borel-Cantelli Lemma, we also have the following corollary.

Corollary 2.7. Suppose that $\alpha>1$. For any $\zeta>0$, the following happens $Q$-almost surely: there exists $N=N\left(\omega_{p}\right)$ such that for any $n \geq N$ and any minimizing path $\pi_{n}$ for $T_{n}\left(\omega_{p}\right)$,

$$
\max _{1 \leq i \leq n} \Delta \pi_{n}(i) \leq n^{\zeta} .
$$


2.4. Concentration of the free energy. In this subsection, we prove Theorem 1.5. By the relation

$$
\log Z_{n}^{\eta, \beta}=\beta n+\log Z_{n}^{1-\eta,-\beta},
$$

we have only to consider the case $\beta \in[-\infty, 0]$. Note that, in this case, $Z_{n}^{\eta, \beta} \leq 1$ $Q$-a.s. For simplicity of notation, we write $Z_{n}(\omega)$ instead of $Z_{n}^{\beta, \eta}$ where $\omega$ is defined as

$$
\omega:=\sum_{(k, x) \in \mathbb{N} \times \mathbb{Z}^{d}}(1-\eta(k, x)) \delta_{(k, x)} .
$$

We write $M:=1+\alpha^{-1}$. For a given configuration $\eta$, or equivalently $\omega$, and an $n$-path $\gamma$, we denote the free energy per path by

$$
F_{n}(\gamma ; \omega):=c_{2} \sum_{i=1}^{n} \Delta \gamma_{i}^{\alpha}-\beta \sum_{i=1}^{n} \eta(i, \gamma(i))=c_{2} t(\gamma)-\beta H_{n}^{\eta}(\gamma)
$$

where the constant $c_{2}$ comes from (1.1).

We assume that $\gamma$ starts at the origin throughout this subsection. Then we can write the partition function as

$$
Z_{n}(\omega)=c_{1}^{n} \sum_{\gamma} e^{-F_{n}(\gamma ; \omega)} .
$$

We define $\bar{\omega}$ by (2.17) and the restricted partition function by

$$
\tilde{Z}_{n}(\omega):=c_{1}^{n} \sum_{\gamma: t(\gamma) \leq n^{1+2 \alpha \theta}} e^{-F_{n}(\gamma ; \omega)} .
$$

First, we bound the difference of partition functions $Z_{n}(\omega)$ and $Z_{n}(\bar{\omega})$. We begin with the following tail bounds.

Lemma 2.8. The following results hold:

(i) There exists $C_{0}>0$ independent of $p \in(0,1]$ such that for all $n \in \mathbb{N}$ and $m>C_{0} n$,

$$
Q\left(T_{n}\left(\omega_{p}\right)>m\right) \leq \exp \left\{-m^{1 \wedge \frac{d}{\alpha}} / C_{0}\right\}
$$

(ii) There exist $c>0$ and $N \in \mathbb{N}$ such that for all $n>N$,

$$
Z_{n}(\omega)-\tilde{Z}_{n}(\omega) \leq e^{-c n^{1+2 \alpha \theta}} .
$$

Proof: (i) The proof is exactly the same as in Lemma 3.2 in Comets et al. (2015). We skip the details.

(ii) We bound $c_{1}^{-n}\left(Z_{n}(\omega)-\tilde{Z}_{n}(\omega)\right)$ from above as follows:

$$
\begin{aligned}
c_{1}^{-n}\left(Z_{n}(\omega)-\tilde{Z}_{n}(\omega)\right) & =\sum_{t(\gamma)>n^{1+2 \alpha \theta}} e^{-F_{n}(\gamma ; \omega)} \\
& =\sum_{k \geq 2} \sum_{\gamma: n^{1+k \alpha \theta}<t(\gamma) \leq n^{1+(k+1) \alpha \theta}} e^{-c_{2} t(\gamma)} \\
& \leq \sum_{k \geq 2} \sum_{\gamma: t(\gamma) \leq n^{1+(k+1) \alpha \theta}} e^{-c_{2} n^{1+k \alpha \theta}} .
\end{aligned}
$$


If $t(\gamma) \leq n^{1+(k+1) \alpha \theta}$, then $\gamma$ has no jump larger than $n^{\frac{1}{\alpha}+(k+1) \theta}$ and thus

$$
\max _{1 \leq i \leq n}|\gamma(i)|_{1} \leq n^{M+(k+1) \theta} .
$$

This yields the bound

$$
\#\left\{\gamma: t(\gamma) \leq n^{1+(k+1) \alpha \theta}\right\} \leq n^{d(M+(k+1) \theta) n} .
$$

Substituting this into (2.23), for sufficiently large $n$, we obtain

$$
Z_{n}(\omega)-\tilde{Z}_{n}(\omega) \leq c_{1}^{n} \sum_{k \geq 2} n^{d(M+(k+1) \theta) n} e^{-c_{2} n^{1+k \alpha \theta}} \leq e^{-c n^{1+2 \alpha \theta}}
$$

with some small constant $c>0$.

We define $\bar{\omega}$ as in (2.17).

Lemma 2.9. For any $q \in[0,1)$ and $\theta>0$, there exist $\lambda, c>0$ and $N \in \mathbb{N}$ such that for any $n>N$ and $p \in[0, q)$,

(i) $Q\left(\left|\log Z_{n}(\omega)-\log Z_{n}(\bar{\omega})\right|>\log 2\right) \leq e^{-c n^{\lambda}}$,

(ii) $0 \leq Q\left[\log Z_{n}(\bar{\omega})\right]-Q\left[\log Z_{n}(\omega)\right] \leq 1$.

Proof: Since $Z_{n}(\omega) \geq c_{1}^{n} e^{-c_{2} s_{p}^{-\alpha} T_{n}\left(\omega_{p}\right)}$, Lemma 2.8-(i) and $Z_{n}(\omega) \leq 1$ imply that there exist $C_{1}, C_{2}>0$ depending on $q$ such that

$$
\begin{aligned}
& Q\left(Z_{n}(\omega) \leq c_{1}^{n} e^{-c_{2} n^{1+\alpha \theta}}\right) \leq e^{-C_{1} n^{\left(1 \wedge \frac{d}{\alpha}\right)(1+2 \alpha \theta)}}, \\
& Q\left[\left|\log Z_{n}(\omega)\right|^{2}\right] \leq C_{2} n^{2}
\end{aligned}
$$

Also it is plain to see (from Lemma 3.3 in Comets et al., 2015) that there exists $C_{3}>0$ such that

$$
Q\left(\omega=\bar{\omega} \text { on }\left[-n^{M+2 \theta}, n^{M+2 \theta}\right]^{d} \times[0, n]\right) \leq e^{-C_{3} n^{d \theta}} .
$$

Thus to prove (i), it suffices to show $Z_{n}(\bar{\omega}) \leq 2 Z_{n}(\omega)$ under the two conditions: $Z_{n}(\omega) \geq c_{1}^{n} e^{-c_{2} n^{1+\alpha \theta}}$ and $\omega=\bar{\omega}$ on $\left[-n^{M+2 \theta}, n^{M+2 \theta}\right]^{d} \times[0, n]$. Observe that $Z_{n}(\omega) \leq Z_{n}(\bar{\omega})$ and that if $\gamma$ exits from $\left[-n^{M+2 \theta}, n^{M+2 \theta}\right]^{d} \times[0, n]$, then $t(\gamma)>$ $n^{1+2 \alpha \theta}$ as it must contain a jump larger than $n^{\frac{1}{\alpha}+2 \theta}$. Therefore under the above conditions, by Lemma 2.8-(ii), there exists $C_{4}>0$ such that

$$
\begin{aligned}
Z_{n}(\bar{\omega})-Z_{n}(\omega) & \leq c_{1}^{n} \sum_{\gamma: \max _{1 \leq k \leq n}|\gamma(k)|_{\infty}>n^{M+2 \theta}} e^{-F_{n}(\gamma ; \omega)} \\
& \leq Z_{n}(\omega)-\tilde{Z}_{n}(\omega) \\
& \leq e^{-C_{4} n^{1+2 \alpha \theta}} \leq Z_{n}(\omega) .
\end{aligned}
$$

This in turn implies

$$
\begin{aligned}
& Q\left[\log Z_{n}(\bar{\omega})\right]-Q\left[\log Z_{n}(\omega)\right] \\
& \leq Q\left(Z_{n}(\omega) \geq c_{1}^{n} e^{-c_{2} n^{1+\alpha \theta}} \text { and } \omega=\bar{\omega} \text { on }\left[-n^{M+2 \theta}, n^{M+2 \theta}\right]^{d} \times[0, n]\right) \log 2 \\
& \quad+Q\left[\left|\log Z_{n}(\omega)\right| ; Z_{n}(\omega)<c_{1}^{n} e^{-c_{2} n^{1+\alpha \theta}} \text { or } \omega \neq \bar{\omega} \text { on }\left[-n^{M+2 \theta}, n^{M+2 \theta}\right]^{d} \times[0, n]\right] \\
& \leq 1,
\end{aligned}
$$

where in the last line we have used (2.24), (2.25) and the Schwarz inequality to bound the second term. 
From Lemma 2.9, once we show the concentration for $\bar{\omega}$, i.e. for any $\delta>0$ there exist $\lambda \in(0,1)$ and $C_{0}, C_{1}>0$ such that

$$
Q\left(\left|\log Z_{n}(\bar{\omega})-Q\left[\log Z_{n}(\bar{\omega})\right]\right|>n^{\frac{1}{2}+\delta}\right) \leq C_{0} e^{-C_{1} n^{\lambda}},
$$

the concentration for $\omega$ immediately follows. We will use the entropy method as in the FPP case and the following two lemmas correspond to Lemma 2.5 and 2.6.

Lemma 2.10. Let $s \in\{1, \cdots, n\}$. We take configurations $\omega, \omega^{\prime}$ such that $\left.\omega\right|_{\{\ell\} \times \mathbb{R}^{d}}=\left.\omega^{\prime}\right|_{\{\ell\} \times \mathbb{R}^{d}}$ for any $\ell \neq s$. Then, for any $n$-path $\gamma$, there exists $\gamma^{\prime}$ such that one of the following holds:

(I) for any $i \neq s, \gamma(i)=\gamma^{\prime}(i)$, and $F_{n}\left(\gamma^{\prime} ; \bar{\omega}^{\prime}\right) \leq c_{2} n^{\zeta(\alpha-1)_{+}+3 \theta}+F_{n}(\gamma ; \bar{\omega})$ or

(II) there exists $k>0$ such that for any $i \notin[s, s+k], \gamma(i)=\gamma^{\prime}(i)$, and

$$
F_{n}\left(\gamma^{\prime} ; \bar{\omega}^{\prime}\right)+c_{2}(k+1) n^{\theta} \leq F_{n}(\gamma ; \bar{\omega})
$$

where the constant $c_{2}$ comes from (1.1) and $(\alpha-1)_{+}=(\alpha-1) \vee 0$.

Proof: When $\alpha \leq 1$, it is easy to prove that (I) holds for any $\gamma$. Suppose that $\alpha>1$ and we will apply the Lemma 2.3 with $\omega^{\prime}=\bar{\omega}^{\prime}$. It suffices to show that (i) and (ii) in Lemma 2.3 imply (I) and (II) in Lemma 2.10, respectively. When (i) holds, we construct a polymer $\gamma^{\prime}$ such that $\gamma^{\prime}(i)=\gamma(i)$ for $i \neq s,\left.\left(s, \gamma^{\prime}(s)\right) \in \bar{\omega}^{\prime}\right|_{\{m\} \times \mathbb{R}^{d}}$, and $\left|\gamma^{\prime}(s)-\gamma(s)\right|_{1} \leq d n^{\theta}$. Then, it is trivial that $\gamma^{\prime}$ satisfies condition (I). On the other hand, we can easily check that (ii) leads to (II) because of the assumption of $\beta \in[-\infty, 0]$, which is declared at the beginning of this subsection.

Lemma 2.11. Let $\omega$ and $\omega^{\prime}$ be the configurations as in Lemma 2.10. For any $\delta>0$, there exist $\theta>0$ and $C>0$ such that for any $n \in \mathbb{N}$,

$$
\left|\log Z_{n}(\bar{\omega})-\log Z_{n}\left(\bar{\omega}^{\prime}\right)\right| \leq C n^{\delta} .
$$

Proof: Note first that for any $\omega$,

$$
\tilde{Z}_{n}(\bar{\omega}) \geq c_{1}^{n} e^{-c_{2} T_{n}(\bar{\omega})} \geq c_{1}^{n} e^{-c_{2} d^{\alpha} n^{1+\alpha \theta}}
$$

since $\bar{\omega}$ has $\theta$-property. This together with Lemma 2.8-(ii) allows us to replace $Z_{n}$ by $\tilde{Z}_{n}$ in the claim. For a path $\gamma^{\prime}$ and $k \in \mathbb{N}$, we define the following sets:

$$
\begin{aligned}
& \Phi_{1}\left(\gamma^{\prime}\right):=\left\{\gamma: t(\gamma) \leq c_{2} n^{1+2 \alpha \theta},\left(\gamma, \gamma^{\prime}\right) \text { satisfies condition (I) }\right\} \\
& \Phi_{2, k}\left(\gamma^{\prime}\right):=\left\{\gamma: t(\gamma) \leq c_{2} n^{1+2 \alpha \theta},\left(\gamma, \gamma^{\prime}\right) \text { satisfies condition (II) with } k\right\}
\end{aligned}
$$

where (I) and (II) are those in Lemma 2.10. Then any $n$-path $\gamma$ lies in one of the above sets and

$$
\begin{aligned}
\tilde{Z}_{n}(\bar{\omega}) & \leq c_{1}^{n} \sum_{\gamma^{\prime}}\left\{\sum_{\gamma \in \Phi_{1}\left(\gamma^{\prime}\right)} e^{-F_{n}(\gamma ; \bar{\omega})}+\sum_{k \geq 1} \sum_{\gamma \in \Phi_{2, k}\left(\gamma^{\prime}\right)} e^{-F_{n}(\gamma ; \bar{\omega})}\right\} \\
& \leq c_{1}^{n} \sum_{\gamma^{\prime}} e^{-F_{n}\left(\gamma^{\prime} ; \bar{\omega}^{\prime}\right)}\left\{\sum_{\gamma \in \Phi_{1}\left(\gamma^{\prime}\right)} e^{c_{2} n^{\zeta(\alpha-1)++3 \theta}}+\sum_{k \geq 1} \sum_{\gamma \in \Phi_{2, k}\left(\gamma^{\prime}\right)} e^{-c_{2} k n^{\theta}}\right\} \\
& \leq c_{1}^{n} \sum_{\gamma^{\prime}} e^{-F_{n}\left(\gamma^{\prime} ; \bar{\omega}^{\prime}\right)}\left\{\left|\Phi_{1}\left(\gamma^{\prime}\right)\right| e^{c_{2} n^{\zeta(\alpha-1)++3 \theta}}+\sum_{k \geq 1}\left|\Phi_{2, k}\left(\gamma^{\prime}\right)\right| e^{-c_{2} k n^{\theta}}\right\} .
\end{aligned}
$$


Since for any $\gamma^{\prime},\left|\Phi_{1}\left(\gamma^{\prime}\right)\right| \leq n^{2 d(M+2 \theta)}$ and $\left|\Phi_{2, k}\left(\gamma^{\prime}\right)\right| \leq n^{2 d(M+2 \theta) k}$ (recall the argument (2.23)), this is further bounded from above by

$$
\begin{aligned}
& c_{1}^{n} \sum_{\gamma^{\prime}} e^{-F_{n}\left(\gamma^{\prime} ; \bar{\omega}^{\prime}\right)}\left\{n^{2 d(M+2 \theta)} e^{c_{2} n^{\zeta(\alpha-1)}++3 \theta}+\sum_{k \geq 1} n^{2 d k(M+2 \theta)} e^{-c_{2} k n^{\theta}}\right\} \\
\leq & c_{1}^{n} \sum_{\gamma^{\prime}} e^{-F_{n}\left(\gamma^{\prime} ; \bar{\omega}^{\prime}\right)}\left\{n^{2 d(M+2 \theta)} e^{c_{2} n^{\zeta(\alpha-1)++3 \theta}}+\sum_{k \geq 1} e^{-c_{2} k n^{\theta} / 2}\right\} \\
\leq & c_{1}^{n} e^{2 c_{2} n^{\zeta(\alpha-1)++3 \theta}} \sum_{\gamma^{\prime}} e^{-F_{n}\left(\gamma^{\prime} ; \bar{\omega}^{\prime}\right)}=e^{2 c_{2} n^{\zeta(\alpha-1)}+3 \theta} Z_{n}\left(\bar{\omega}^{\prime}\right) .
\end{aligned}
$$

With the symmetry between $\omega$ and $\omega^{\prime}$, this implies

$$
\begin{aligned}
\left|\log Z_{n}(\bar{\omega})-\log Z_{n}\left(\bar{\omega}^{\prime}\right)\right| & \leq \log \left\{2 e^{2 c_{2} n^{\zeta(\alpha-1)_{+}+3 \theta}}\right\} \\
& \leq 3 c_{2} n^{\zeta(\alpha-1)_{+}+3 \theta} .
\end{aligned}
$$

If we take $\zeta>0$ sufficiently small so that $3 c_{2} n^{\zeta(\alpha-1)_{+}+3 \theta}<n^{\delta}$, the proof is completed.

Thanks to Lemma 2.11, we can use Theorem 6.7 in Boucheron et al. (2013) and we get the desired concentration (2.26) of $\log Z_{n}(\bar{\omega})$.

\section{Non-random fluctuation}

3.1. FPP Case. In this subsection, we deduce the so-called non-random fluctuation bound (1.7) from the concentration bound (1.6). This is a well-studied subject in the theory of first passage percolation and we shall adapt the argument of Zhang (2010) to our setting.

Proof of (1.7): Let $\chi>1 / 2, M=1+\alpha^{-1}$ and $\pi_{n}=\pi_{n}^{(n)}$, i.e. a minimizing path for $T_{n}\left(\omega_{p}\right)$. We define a face to face passage time

$$
\Phi_{n}\left(k, l ; \omega_{p}\right)=\inf \left\{\sum_{i=k+1}^{l}\left|x_{i-1}-x_{i}\right|_{1}^{\alpha}:\left|x_{k}\right|_{\infty}<n^{M} \text { and }\left\{\left(i, x_{i}\right)\right\}_{k+1}^{l} \subset \omega_{p}\right\}
$$

and introduce the events

$$
\begin{aligned}
& \mathcal{A}_{1}^{\theta}(n)=\left\{\omega_{p}=\bar{\omega}_{p} \text { on }[0,2 n] \times\left[-n^{M}, n^{M}\right]^{d}\right\} \\
& \mathcal{A}_{2}(n)=\left\{\begin{array}{c}
\text { There exist }|x|_{\infty} \leq 1 / 2 \text { and a minimizing path for } \\
\Phi_{n}\left(n, 2 n ; \omega_{p}\right) \text { starting at }(n, x) .
\end{array}\right\} .
\end{aligned}
$$

Lemma 3.1. We fix a sufficiently small constant $\theta>0$. Then, there exists $c>0$ such that for all sufficiently large $n$,

(i) $Q\left(\mathcal{A}_{1}^{\theta}(n)\right) \geq 1-\exp \left\{-c n^{\theta}\right\}$,

(ii) $Q\left(\mathcal{A}_{2}(n)\right) \geq 2^{-d} n^{-d M}$.

Proof: The first statement follows from the proof of Lemma 3.3 in Comets et al. (2015). Since the law of $\omega_{p}$ is invariant under time-space shift, the probabilities

$Q$ (There exists a minimizing path for $\Phi_{n}\left(0, n ; \omega_{p}\right)$

$$
\text { starting at } \left.(0, x) \text { with }|x-y|_{\infty} \leq 1 / 2\right)
$$


for $y \in \mathbb{Z}^{d} \cap\left(-n^{M}, n^{M}\right)^{d}$ are the same as $Q\left(\mathcal{A}_{2}(n)\right)$. Since the union of the above events has probability one and the number of possible $y$ 's is less than $\left(2 n^{M}\right)^{d}$, the second statement is completed.

With this lemma and (1.6), we can complete the proof of (1.7). Note first that on the event $\mathcal{A}_{1}^{\theta}(n)$, we have $\pi_{2 n}(n) \in\left(-n^{M}, n^{M}\right)^{d}$ as the displacement of $\pi_{2 n}$ until time $n$ is at most $2 n \cdot n^{\theta} \vee(2 n)^{\theta+\alpha^{-1}}<n^{M}$ for any sufficiently small $\theta$. As a result, one has

$$
T_{2 n}\left(\omega_{p}\right) \geq T_{n}\left(\omega_{p}\right)+\Phi_{n}\left(n, 2 n ; \omega_{p}\right)
$$

since the second half of $\pi_{2 n}$ is candidates of the face to face minimizing paths. On the other hand, from Lemma 2.5 , for any $\zeta>0$, if we take $\theta>0$ sufficiently small, on the event on $\mathcal{A}=\mathcal{A}_{1}^{\theta}(n) \cap \mathcal{A}_{2}(n)$,

$$
\Phi_{n}\left(n, 2 n ; \omega_{p}\right) \geq T\left(n, 2 n ; \omega_{p}\right)-C n^{\zeta(\alpha-1)_{+}+\theta}
$$

with some $C>0$ independent of $p$ since only possible differences come from the starting points, which can be controlled by using the mean value theorem. Therefore on $\mathcal{A}$, for any $\chi>1 / 2$ and sufficiently large $n \in \mathbb{N}$, we have the following almost super-additivity:

$$
T_{2 n}\left(\omega_{p}\right) \geq T_{n}\left(\omega_{p}\right)+T\left(n, 2 n ; \omega_{p}\right)-n^{\chi} .
$$

Now we use (1.6) to obtain

$$
Q\left(\left|T_{n}\left(\omega_{p}\right)-Q\left[T_{n}\left(\omega_{p}\right)\right]\right|>n^{\chi}\right) \leq C_{1} \exp \left\{-C_{2} n^{\lambda}\right\}
$$

and the same bound for $T_{2 n}\left(\omega_{p}\right)$ and $T_{2 n}\left(\omega_{p}\right)$. These bounds and Lemma 3.1 show that for all sufficiently large $n$,

$$
\mathcal{A} \cap \bigcap_{(k, l) \in\{(0,2 n),(0, n),(n, 2 n)\}}\left\{\left|T\left(k, l ; \omega_{p}\right)-Q\left[T\left(k, l ; \omega_{p}\right)\right]\right| \leq n^{\chi}\right\}
$$

has positive probability and in particular non-empty. Hence we can replace the passage times in (3.1) by their expectation at the cost of extra $-3 n^{\chi}$ on the righthand side to obtain

$$
\frac{1}{2 n} Q\left[T_{2 n}\left(\omega_{p}\right)\right] \geq \frac{1}{n} Q\left[T_{n}\left(\omega_{p}\right)\right]-4 n^{\chi-1} .
$$

Iterating this, we arrive at

$$
\frac{1}{n} Q\left[T_{n}\left(\omega_{p}\right)\right] \leq \frac{1}{2^{k} n} Q\left[T_{2^{k} n}\left(\omega_{p}\right)\right]+4 n^{\chi-1} \sum_{j=1}^{k-1} 2^{(\chi-1) j} .
$$

and letting $k \rightarrow \infty$ proves $Q\left[T_{n}\left(\omega_{p}\right)\right] \leq n \mu_{p}+C n^{\chi}$. Moreover, due to the usual subadditivity, we get the converse inequality $n \mu_{p} \leq Q\left[T_{n}\left(\omega_{p}\right)\right]$. Thus we have (1.7). From (1.6) and (1.7), we obtain (1.8).

3.2. Free energy Case. The proof is almost the same as that for the FPP, so we only mention the outline here. To prove Theorem 1.3, we again use the argument of Zhang. Let

$$
Z(k, \ell, x, \omega):=c_{1}^{n} \sum_{\gamma: \gamma(k)=x} e^{\beta \sum_{i=k+1}^{\ell} \eta(i, \gamma(i))} e^{-c_{2} \sum_{i=k+1}^{\ell} \Delta \gamma(i)^{\alpha}} .
$$


Let $M=1+\frac{1}{\alpha}$ and we define an event $\mathcal{A}$ by

$$
\mathcal{A}:=\left\{Z(n, 2 n, 0, \omega)=\sup _{x \in\left[-n^{M} / 2, n^{M} / 2\right]^{d}} Z(n, 2 n, x, \omega), Z_{2 n}(\omega) \leq 2 \tilde{Z}_{2 n}(\omega)\right\} .
$$

One can show, as in the case of the FPP, that for sufficiently large $n$,

$$
Q(\mathcal{A}) \geq \frac{1}{2 n^{M d}} .
$$

On this event,

$$
\begin{aligned}
& \log Z_{2 n}-\log 2 \leq \log \tilde{Z}_{2 n} \\
& \leq \log \left\{c_{1}^{2 n} \sum_{\substack{\gamma: \max _{1 \leq i \leq n}|\gamma(i)|_{\infty} \leq n^{M} / 2 \\
\gamma(0)=0}} e^{-F_{2 n}(\gamma ; \omega)}\right\} \\
& \leq \log \left\{c_{1}^{n} \sum_{x \in\left[-n^{M} / 2, n^{M} / 2\right]^{d}} \sum_{\substack{\gamma: \gamma(n)=x \\
\gamma(0)=0}} e^{-F_{n}(\gamma ; \omega)} Z(n, 2 n, x, \omega)\right\},
\end{aligned}
$$

where we have used the argument below (2.23) in the second inequality and divided paths at its $n$-th point in the third ineqality. Thanks to the choice of $\mathcal{A}$, this is further bounded from above by

$$
\begin{aligned}
& \log \left\{c_{1}^{n} \sum_{\substack{x \in\left[-n^{M} / 2, n^{M} / 2\right]^{d} \\
\gamma: \gamma(n)=x \\
\gamma(0)=0}} e^{-F_{n}(\gamma ; \omega)} Z(n, 2 n, 0, \omega)\right\} \\
& \leq \log \left(Z_{n}(\omega) Z(n, 2 n, 0, \omega)\right)=\log Z_{n}(\omega)+\log Z(n, 2 n, 0, \omega) .
\end{aligned}
$$

From the concentration (1.9) and this, for any $\chi>1 / 2$, there exists constant $C>0$ independent of $n$ such that,

$$
Q\left[\log Z_{2 n}\right] \leq 2 Q\left[\log Z_{n}\right]+C n^{\chi} .
$$

In a similar way to the proof of Theorem 1.3, we have that for any $k$,

$$
\frac{Q\left[\log Z_{2^{k} n}\right]}{2^{k} n} \leq \frac{Q\left[\log Z_{n}\right]}{n}+C^{\prime} n^{\chi-1}
$$

where $C^{\prime}>0$ is a constant independent of $n$. As $k \rightarrow \infty$, we have

$$
\varphi(p, \beta) \leq \frac{1}{n} Q\left[\log Z_{n}\right]+C^{\prime} n^{\chi-1} .
$$

Finally, we shall derive the converse estimate by a similar way. Indeed, we replace $\mathcal{A}$ by $\left\{Z(n, 2 n, 0, \omega)=\min _{x \in\left[-n^{M} / 2, n^{M} / 2\right]^{d}} Z(n, 2 n, x, \omega), Z_{n}(\omega) \leq 2 \tilde{Z}_{n}(\omega)\right\}$. On this event, we have

$$
\log Z_{2 n} \geq \log \tilde{Z}_{n}+\log Z(n, 2 n, 0, \omega) \geq \log 2+\log Z_{n}+\log Z(n, 2 n, 0, \omega),
$$

which implies

$$
\varphi(p, \beta) \geq \frac{1}{n} Q\left[\log Z_{n}\right]-C^{\prime} n^{\chi-1} .
$$

This yields (1.10). From (1.9) and (1.10), it is immediate to prove (1.11). 


\section{Proof of continuity results}

Thanks to the non-random fluctuation results, it suffices to show the continuities of $Q\left[T_{n}\right]$ and $Q\left[\log Z_{n}\right]$ for fixed $n \in \mathbb{N}$.

Lemma 4.1. For any $n \in \mathbb{N}, Q\left[T_{n}\left(\omega_{p}\right)\right]$ is a continuous function of $p \in[0,1]$.

Proof: Note that $T_{n}(\cdot)$ is continuous on the set of locally finite point configurations with respect to the vague topology. Indeed for any $\omega$, the definition of passage time tells us that only points inside a compact set matter. By local finiteness we can choose the compact set in such a way that its boundary contains no points of $\omega$. Now if $\omega_{N} \rightarrow \omega$ vaguely as $N \rightarrow \infty$, then the points of $\omega_{N}$ inside the compact set converge to those of $\omega$ in the Hausdorff metric and then it easily follows that $T_{n}\left(\omega_{N}\right) \rightarrow T_{n}(\omega)$. By using the Skorohod representation theorem, we may assume that $Q$-almost surely, $\omega_{p} \rightarrow \omega_{p}^{\prime}$ vaguely as $p \uparrow p^{\prime}$. Then by continuity, $T_{n}\left(\omega_{p}\right) \rightarrow T_{n}\left(\omega_{p}^{\prime}\right)$ as $p \uparrow p^{\prime}, Q$-almost surely. From this, $T_{n}\left(\omega_{p}\right)$ is continuous in $p \in[0,1]$. Since the uniform integrability of $\left\{T_{n}\left(\omega_{p}\right)\right\}_{p \in(0,1]}$ follows from Lemma 2.8, $L^{1}(Q)$ convergence follows.

The following lemma can be proved by a similar way to Lemma 4.1.

Lemma 4.2. $Q\left[\log Z_{n}\right]$ is jointly continuous on $(p, \beta) \in[0,1) \times[-\infty, \infty)$.

From Lemmas 4.1 and 4.2, we obtain Corollaries 1.4 and 1.7, respectively.

\section{Acknowledgements}

The author gratefully acknowledges useful discussions with Ryoki Fukushima, especially about the case $\alpha \leq 1$. He also thanks the anonymous referee for the careful reading of the manuscript.

\section{References}

S. Boucheron, G. Lugosi and P. Massart. Concentration inequalities. Oxford University Press, Oxford (2013). ISBN 978-0-19-953525-5. MR3185193.

F. Comets, R. Fukushima, S. Nakajima and N. Yoshida. Limiting results for the free energy of directed polymers in random environment with unbounded jumps. J. Stat. Phys. 161 (3), 577-597 (2015). MR3406700.

R. Fukushima and N. Yoshida. On exponential growth for a certain class of linear systems. ALEA Lat. Am. J. Probab. Math. Stat. 9 (2), 323-336 (2012). MR3069368.

O. Garet, J.-B. Gouéré and R. Marchand. The number of open paths in oriented percolation. Ann. Probab. 45 (6A), 4071-4100 (2017). MR3729623.

C. D. Howard and C. M. Newman. Geodesics and spanning trees for Euclidean first-passage percolation. Ann. Probab. 29 (2), 577-623 (2001). MR1849171.

H. Krug and H. Spohn. Kinetic roughening of growing surfaces. In Solids Far From Equilibrium, pages 479-582. Cambridge University Press (1991). MR1163829.

Q. Liu and F. Watbled. Exponential inequalities for martingales and asymptotic properties of the free energy of directed polymers in a random environment. Stochastic Process. Appl. 119 (10), 3101-3132 (2009). MR2568267. 
Y. Zhang. On the concentration and the convergence rate with a moment condition in first passage percolation. Stochastic Process. Appl. 120 (7), 1317-1341 (2010). MR2639748. 